\title{
Achieving Ultra-Low Detection Limit Using Nanofiber Labels for Rapid Disease Detection
}

\author{
Emmanuel Gikunoo ${ }^{*}$, Adeyabeba Abera ${ }^{2}$, Eyassu Woldesenbet ${ }^{2,3}$ \\ ${ }^{1}$ Materials Engineering Department, Kwame Nkrumah University of Science and Technology, Kumasi, Ghana \\ ${ }^{2}$ Mechanical Engineering Department, National Science Foundation Center for Next Generation Composites, \\ Southern University and A \& M College, Baton Rouge, USA \\ ${ }^{3}$ Mechanical Engineering Department, National Science Foundation Center for Next Generation Composites, \\ Louisiana State University, Baton Rouge, USA \\ Email:
}

Received 21 October 2014; revised 28 November 2014; accepted 23 December 2014

Copyright (C) 2014 by authors and Scientific Research Publishing Inc.

This work is licensed under the Creative Commons Attribution International License (CC BY). http://creativecommons.org/licenses/by/4.0/

(c) (i) Open Access

\section{Abstract}

Early diagnosis of diseases is critical in its effective management. Traditional disease detection methods require specialized equipment and trained personnel. With the introduction of rapid diagnostic test kits (RDTs), disease detection has become easier and faster. However, these RDTs have failed to compete with the specialized laboratory equipment due to their high detection limits and false alarm rates. This paper presents a novel method of using carbon nanofibers (CNFs) grown on glass microballoons (NMBs) to achieve ultra-low detection limits in RDTs. The NMBs have millions of nanosized CNFs grown on each microballoon, with each CNF having a strong bonding affinity for antibodies. The NMBs conjugated with secondary antibodies have therefore a significantly higher probability of capturing minute antigen concentrations in solution. Furthermore, the dark color formation at the capture zone makes visual disease detection possible. Human Immunoglobulin G (IgG) was selected as the model analyte to study the performance of NMBs using a sandwich immunoassay protocol. Ultra-low electrical detection limit of $(4 \mathrm{pg} / \mathrm{ml})$ and rapid response ( $\sim 1$ minute) was achieved using this method.

\section{Keywords}

Rapid Diagnostic Test Kits, Detection Limit, Carbon Nanofibers, Immunoassay, Immunoglobulin G

\footnotetext{
${ }^{*}$ Corresponding author.
} 


\section{Introduction}

Biosensors use specific biochemical reactions mediated by purified enzymes, immunoassay systems, or human cells to detect chemical compounds. These sensors detect the chemical compounds by generating chemical, mechanical, magnetic, electrical, thermal or optical signals as a result of biochemical reactions [1]-[6]. In general, two broad categories of biosensors have been recognized [7]. The first category includes expensive and sophisticated, high-throughput laboratory systems capable of rapid, accurate, and convenient measurement of complex biological components. These laboratory systems require skilled laboratory technicians for their operation. The second category consists of inexpensive and easy-to-use portable devices capable of analyzing biological interactions. Unlike the laboratory systems, these portable devices permit individuals with no clinical training to produce a limited form of biomedical information [7] [8].

The second group, classified as rapid diagnostic test kits (RDTs), are gaining popularity in wide spread use, especially in low-resource settings. RDTs are immunoassay systems that rely on the specific binding of antigens to their primary and secondary antibodies, with the primary antibodies usually being immobilized on solid supports, such as polystyrene or glass substrate [9]. Different types of labels have also been employed in RDTs to assist in visual observations of the test result. The most commonly used labels are colloidal-gold, silver enhanced gold, blue latex beads, and carbon black nanoparticles [10]. These labels are often designed to generate signals for optical detection using colorimetric or fluorescent techniques. Colorimetric detection while simple and cost-effective lacks in the high sensitivity required for early diagnosis [10] [11]. This method is incapable of producing quantitative analysis of the results and is also limited by high false-alarm rates leading to inconclusive or wrong diagnostics [8]. Fluorescent techniques also suffer from photo bleaching and the need for a sophisticated reader device [11]. In addition, an incubation period of at least few minutes is necessary prior to the fluorescence emission. The minimum incubation period currently being reported for RDTs is three minutes [12]. Therefore, there is a growing need to develop RDTs with ultra-low detection limits, faster response time, and zero false-alarm rates. Such RDTs will be able to compete with laboratory diagnostic systems for the early detection of many fatal diseases, such as malaria.

The use of carbon nanostructures such as CNFs, nanotubes (CNTs), or graphene nanosheets, as an alternative labeling agent has the potential to address some of the shortfalls of RDTs. Several demonstrations confirming the possibilities of incorporating CNTs have been reported [11] [13]-[15]. However, the utilization of individual CNTs continues to pose a challenge due to signal repeatability and higher false-alarm rates. Large range of signal fluctuations is encountered due to lack of control in immobilizing consistent number of CNTs to form electron pathways at the capture zone. In addition, the probability of capture of the antibody conjugated CNTs is also low due to size. Consequently, the signals generated fluctuate resulting in misleading diagnosis. Nevertheless, if carbon nanostructures can be organized on a micro-sized particle the immobilization of consistent microparticles-carbon nanostructures conjugate would be possible. Therefore, consistent electron pathways would be formed and signal fluctuations could be minimized. Such structure would also provide ultra-low detection limits, since immobilization of one of these microparticles is practically equal to capturing millions of CNTs/CNFs. This method would also significantly amplify the generated detection signal.

This paper demonstrates the successful implementation of this method in immunosensing. CNFs grown on glass microballoons (NMBs) were utilized as a novel label in RDTs. Human Immunoglobulin G (IgG) was selected as model analyte for this study. The NMBs conjugated with IgG were prepared and used to detect antiIgG with different concentrations ranging from $0.002 \mathrm{ng} / \mathrm{ml}$ to $50 \mathrm{ng} / \mathrm{ml}$. The following sections explain the details of the preparation of this immunosensor as well as the investigation of its performance and detection limit. Similar tests were repeated in the same settings but with individual CNTs as label to accurately investigate the actual improvement in detection limit when NMBs were applied.

\section{Materials and Methods}

\subsection{Materials}

S22 glass microballoons were purchased from 3M Corporation, USA. The following are the chemicals used for the immobilization of the IgG on NMBs: Polyethylene glycol solution (PEG) (MW 8000) was purchased from Sigma-Aldrich, GA, USA; phosphate buffered saline 1X solution (PBS) obtained from Thermo Fisher Scientific, IL, USA; purified immunoglobulin G (IgG) from human serum, IgG-Fluorescein isothiocyanate isomer I (FITC) 
from human serum, and anti-human IgG antibody produced in goat were obtained from Sigma-Aldrich, MO, USA. All IgG contained samples were obtained in a solution of $0.01 \mathrm{M}$ phosphate buffered saline, $\mathrm{pH} 7.4$ and contains $15 \mathrm{mM}$ of sodium azide. Micro glass slides used for the study were obtained from Corning, NY, USA.

\subsection{Functionalization of CNFs on the NMBs}

CNFs were first grown on Ni-coated glass microballoons using a technique described in reference [16]. Introduction of carboxyl functional groups on the CNFs was achieved by oxidizing the NMBs in air at $400^{\circ} \mathrm{C}$ in a tube furnace for an hour.

\subsection{Preparation of NMB-IgG Conjugates}

Covalent conjugation of antibodies with CNFs required a two-step reaction approach in preparing the surface of CNFs. The approach described in an earlier work [17] was used in preparing the surface of CNFs for covalent conjugation with IgG. The process introduced semi-stable amine-reactive sulfo-NHS ester intermediate which helped increase the solubility and stability of the NMBs in solution. The treated NMBs were further coated with PEG. The PEG coating was applied to prevent nonspecific binding (NSB) of unwanted proteins to the NMBs. The ester intermediate on the NMBs then reacted with the primary amine group on the surfaces of the IgG, forming very strong amide bonds in the process [18]. Successful competitive and sandwich immobilization protocols were confirmed using IgG and anti-IgG tagged with FITC fluorochrome.

\subsection{Preparation of the Glass Substrate}

Glass slides, used as substrates for the immunosensor, were cut into rectangular shapes $(1 \mathrm{~cm} \times 2.5 \mathrm{~cm})$ and capture zones were marked within the mid portion of the slides. Slides were thoroughly cleaned and activated with amino functional groups as described previously [17]. Details of immobilizing IgG on glass slides to capture antiIgG based on sandwich immunoassay protocol as described in reference [17]. All slides were finally incubated in a $2 \%$ BSA and $0.05 \%$ Tween-20 in PBS solution for an hour in order to block all capture zones from NSB. A plain glass slide was also incubated in the 2\% BSA and 0.05\% Tween-20 in PBS solution for an hour. This was used as a control test for NSB.

\subsection{Preparation of the Immunosensor}

Anti-IgG $(0.01 \mathrm{ng} / \mathrm{ml}$ in PBS) was applied onto the capture zone of the treated slide. The slide was allowed to incubate for 30 seconds after which it was thoroughly rinsed in PBS solution. $1 \mathrm{mg}$ of IgG conjugated NMBs were then suspended in $5 \mathrm{ml}$ PBS solution. The prepared slide was then dipped in the NMB/PBS solution for 30 seconds to form the reaction. Similar immobilization process of NMBs was repeated for anti-IgG concentrations of $0.002,0.004,0.008,0.1,0.5,1,5,10$ and $50 \mathrm{ng} / \mathrm{ml}$. Visual observation and electrical resistivity measurements were performed on the slides to study the detection limit.

\subsection{Characterization}

Scanning electron microscopy (SEM), optical microscopy, electrical resistivity characterization, and percent area covered of NMBs on the capture zone were performed. The procedure and equipment for the characterization was reported in an earlier study [17].

\section{Results and Discussion}

\subsection{Preparation of NMBs and Antibody Conjugation}

The successful growth of CNFs on microballoons is depicted in Figure 1(a). Several millions of the nanosized CNFs were organized on each microballoon. Average NMB sizes of $25 \mu \mathrm{m}$ were grown. These hair-like structures, with high aspect ratio and high chemical affinity, were potential sites on which antibodies could be immobilized.

The oxidation of NMBs in air led to the grafting of the carboxyl functional groups onto the surface of the CNFs. The presence of large amounts of amine on the surface of antibodies permitted their intermolecular con- 
jugation with the carboxylated CNFs on NMBs through an amidation process [19] [20]. Sandwiched conjugation of IgG/anti-IgG/IgG-FITC with NMBs generated fluorescence signals proving successful conjugation of NMBIgG (Figure 1(b)).

NSB of IgG to the NMBs was addressed by a similar fluorescence study. The surfaces of NMB-IgG conjugates were coated with PEG. The formation of a polyethylene oxide chains resulted in a highly hydrophilic, neutrally charged, sterically hindering layer at the surfaces of the NMBs [11] [21] [22]. The PEG-coated NMB-IgG conjugates were reacted by IgG-FITC. There was no fluorescent signal observed on these conjugates verifying the successful NSB of IgG to NMBs. The effective NSB therefore minimizes any false alarm from the system.

\subsection{Visual Detection}

Successful detection of anti-IgG on the glass slides were achieved within one minute using the procedure above. This was detected by observing visible network of NMBs on the capture zone. The use of NMBs with several thousands of conjugated antibodies in this immunosensor significantly increased the probability of antigen entrapment. The captured NMBs were bond tightly to the substrate due to the strong interlocking mechanism between antigens and antibodies. Increasing anti-IgG concentration increased the density of the captured NMBs as depicted in the illustration (Figures 2(a)-(c)). The network formed by the immobilized NMBs at the capture zone is fairly visible with the naked eye at concentrations up to $0.1 \mathrm{ng} / \mathrm{ml}$ of anti-IgG.

An optical micrograph was used to observe the arrangement of the NMB networks at the capture zone. Figure 2(d) shows the micrograph of the NMBs captured on the glass slide in a sandwich assay format with anti-IgG concentration of $0.1 \mathrm{ng} / \mathrm{ml}$. The NMBs are seen to be individually interlocked but in close contact with each other on the glass slide. This forms an interconnecting conducting pathway for an effective electron transfer during resistivity measurements. Increasing the anti-IgG concentration resulted in a denser network of NMBs within the capture zone allowing electrons to flow through them much easier. Electrical resistivity of the zone therefore decreases with increasing antigen concentration.

Optical micrographs of NMBs immobilized on the capture zone for the various anti-IgG concentrations were also obtained. ImageJ software was used to determine the percentage area covered by immobilized NMBs on the various micrographs. A linear relationship between the number of immobilized NMBs and anti-IgG concentrations was observed as shown in Figure 3(a) $\left(R^{2}\right.$ value $\left.=0.9981\right)$.

\subsection{Electrical Detection}

In order to confirm the visual results, electrical resistivity measurements of the capture zone were performed. Four-point probe method was used for resistivity measurement to eliminate the impedance contribution of the wiring and contact resistances. A plot of the resistivity measurements obtained against concentration of anti-IgG is shown in Figure 3(b). As can be observed in the figure, subsequent decline in the resistivity within the capture zone was observed when the zone was exposed to a suspension containing higher anti-IgG concentration.

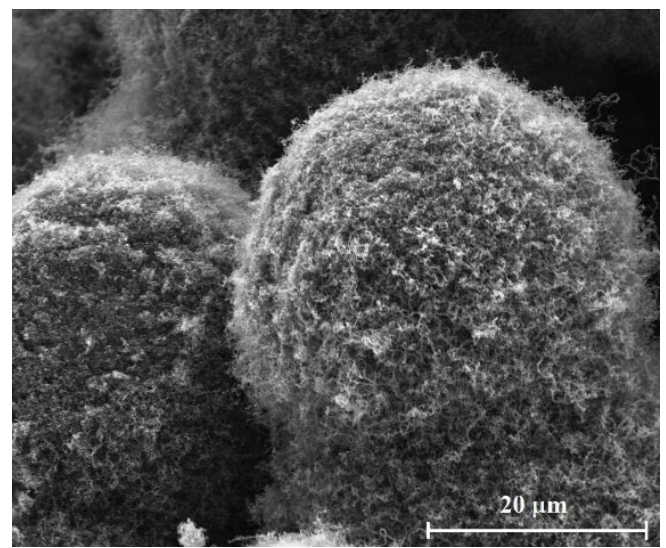

(a)

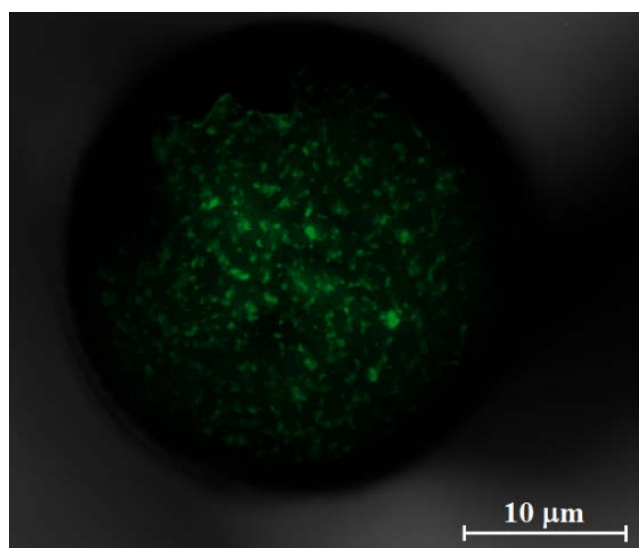

(b)

Figure 1. (a) SEM micrograph of as-fabricated NMBs; and (b) Fluorescence micrograph showing successful sandwich conjugation of IgG/anti-IgG/IgG-FITC on NMB. 


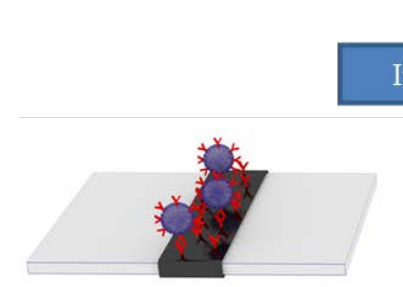

(a)

Increasing anti- $\operatorname{IgG}$ concentration

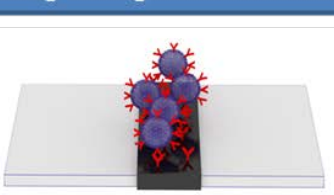

(b)

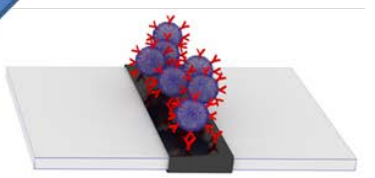

(c)

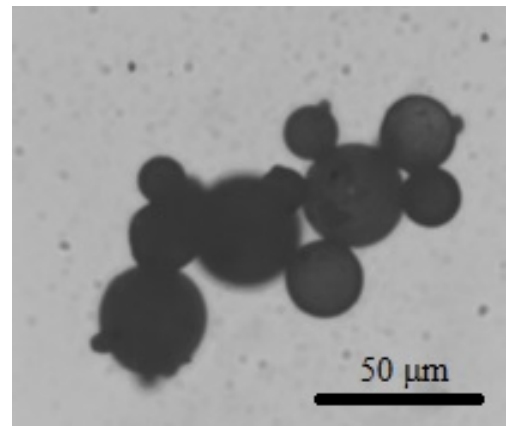

(d)

Figure 2. (a)-(c) Schematic of increasing anti-IgG concentrations on NMBs network at the capture zone; and (d) Optical micrograph showing NMBs captured on glass slide in a sandwich assay format with $0.1 \mathrm{ng} / \mathrm{ml}$ of anti-IgG.

This was attributed to the formation of interconnecting conducting pathway due to the capture of more NMBs. At anti-IgG concentration of $0.002 \mathrm{ng} / \mathrm{ml}$, the resistivity obtained was the same as that of the plain glass. Change in resistivity was first observed at $0.004 \mathrm{ng} / \mathrm{ml}$ hence the detection limit of anti-IgG using NMBs. At anti-IgG concentrations above $5 \mathrm{ng} / \mathrm{ml}$ resistivity gradually started to reach a plateau. At higher concentrations (>10 ng/ml) saturation occurred. Hence, further increases in the number of captured NMBs did not result in any significant changes in the resistivity.

The resistivity measurement of the NSB test was obtained to be $8.74 \times 1010 \Omega$-cm. This compared favorably with the resistivity obtained for the plain glass slides. The successful elimination of NSB reduced the generation of false positives and hence minimization of false alarm.

Nonlinear regression methods were used to fit a curve to the data points. This provided a function for measured resistivity based on antigen concentration as the independent variable. The following function provided a good agreement with the experimental data $\left(R^{2}=0.9552\right)$ :

$$
\operatorname{Ln}(R)=a \cdot \exp (-b \cdot C)+R_{0}
$$

In this function, $R$ and $C$ are resistivity $(\Omega \cdot \mathrm{cm})$ and antigen concentration $(\mathrm{ng} / \mathrm{ml})$, respectively, and $a, b$, and $R_{0}$ are constants. The calculated values for the constants are presented in Table 1 . Measuring the resistivity during any diagnostics can be used to predict the level of disease causing antigen in a sample. This will provide healthcare professionals the needed information for the management of the disease.

\subsection{Comparison of NMB-Labels with Individual CNT-Labels}

This comparison study was based on an earlier findings by Abera et al. using individual CNTs as label [11]. To highlight the signal amplification potential that NMBs are offering for low detection limit, similar tests were repeated using individual CNTs on our immunosensor. As shown in Figure 4, the change in measured resistivity was significant when NMBs were used as labels, making them very sensitive to detection of even slight changes in concentration of antigens. In the case of $50 \mathrm{ng} / \mathrm{ml}$ of anti-IgG, the measured resistivity of immobilized CNTs was $\sim 3.45$ times less compared to baseline $(9.30 \times 1010 \Omega-\mathrm{cm})$. The resistivity values obtained from immobilized CNTs were very close to the baseline. A distinguishable limit of $0.01 \mathrm{ng} / \mathrm{ml}$ was obtained for the CNTs. Below this limit, all measurements were approximately equal to the baseline. With the immobilized NMBs, the limit was obtained at a lower IgG concentration of $0.004 \mathrm{ng} / \mathrm{ml}$. It can also be seen that with the use of NMBs 


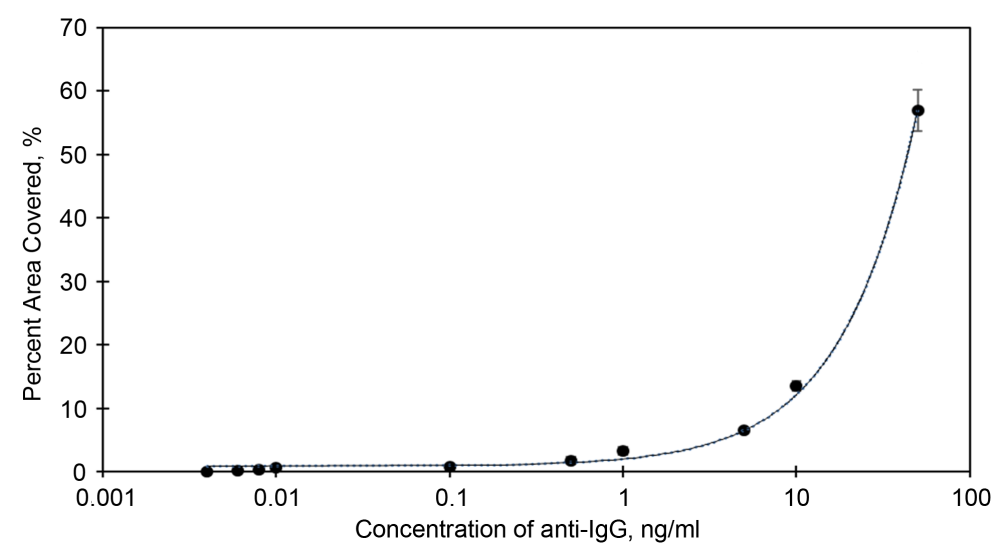

(a)

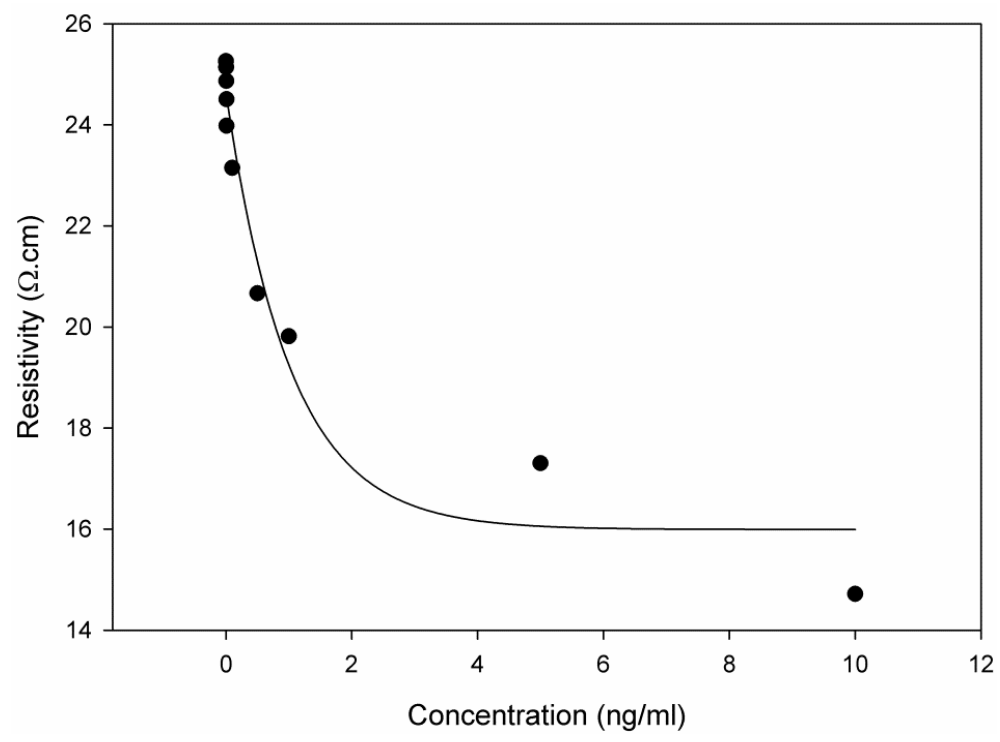

(b)

Figure 3. (a) Variation of percent area of the capture zone covered with NMBs against anti-IgG concentrations; and (b) Variation of the resistivity of the capture zone with anti-IgG concentration.

Table 1. Calculated coefficients for the conducted tests.

\begin{tabular}{cccc}
\hline & Coefficient & Standard Error & CV (\%) \\
\hline$R_{0}$ & 14.8149 & 0.7196 & $4.85 \%$ \\
$a$ & 9.7270 & 0.8485 & $8.72 \%$ \\
$b$ & 0.7039 & 0.2275 & $32.31 \%$ \\
\hline
\end{tabular}

significant variation in the resistivity with concentration was obtained. This would enable the health professional to accurately determine the severity or progression of the disease in a patient.

The ability of detecting very low concentration of disease utilizing the NMBs was even comparable to some of the more sophisticated methods which have been suggested to replace the conventional enzyme-linked immunosorbent assay (ELISA) technique (Table 2). This ultra-low disease detection stems from the fact that the millions of CNFs grown on each microballoon makes it possible for several thousands of antibodies to bind with each NMB. Large numbers of antibodies with fewer labels obviously have higher chance of interlocking with immobilized antigens than individually suspended CNTs. 
Table 2. Reported detection limit of human IgG using different immunoassay-based approaches.

\begin{tabular}{|c|c|c|c|c|}
\hline Label & Signal detection & Detection limit (ng/mL) & Disadvantage & Ref. \\
\hline $\begin{array}{c}\text { Ferrocene-tagged peptide } \\
\text { nanowired coated with AuNP }\end{array}$ & Electro-chemistry & $5 \times 10^{-6}$ & Rather complex instrumentation & [23] \\
\hline $\begin{array}{l}\text { CdSe quantum dots with } \\
\text { carbon nanotubes and AuNPs }\end{array}$ & ECL & 0.0006 & $\begin{array}{l}\text { Rather complex instrumentation/QD } \\
\text { are toxic materials }\end{array}$ & [24] \\
\hline NMBs & Electrical & 0.004 & & \\
\hline Gold nanoclusters (AuNCs) & & 0.005 & 5 minsincubation time & [25] \\
\hline $\begin{array}{l}\text { Carbon nanotubes coated with } \\
\text { palladium nano-particles }\end{array}$ & Electro-chemistry & 0.044 & $\begin{array}{l}\text { Rather complex instrumentation/Higher } \\
\text { detection limit }\end{array}$ & [26] \\
\hline AuNP-graphene nanosheets & Electro-chemistry & 0.05 & $\begin{array}{l}\text { Rather complex instrumentation/Higher } \\
\text { detection limit }\end{array}$ & [27] \\
\hline CNTs & Electro-chemistry & 0.1 & $\begin{array}{l}\text { Rather complex instrumentation/Higher } \\
\text { detection limit }\end{array}$ & [28] \\
\hline Amorphous carbon nano-particles & Chemi-luminescence & 1.9 & $\begin{array}{l}\text { Rather complex instrumentation/Higher } \\
\text { detection limit }\end{array}$ & [29] \\
\hline
\end{tabular}

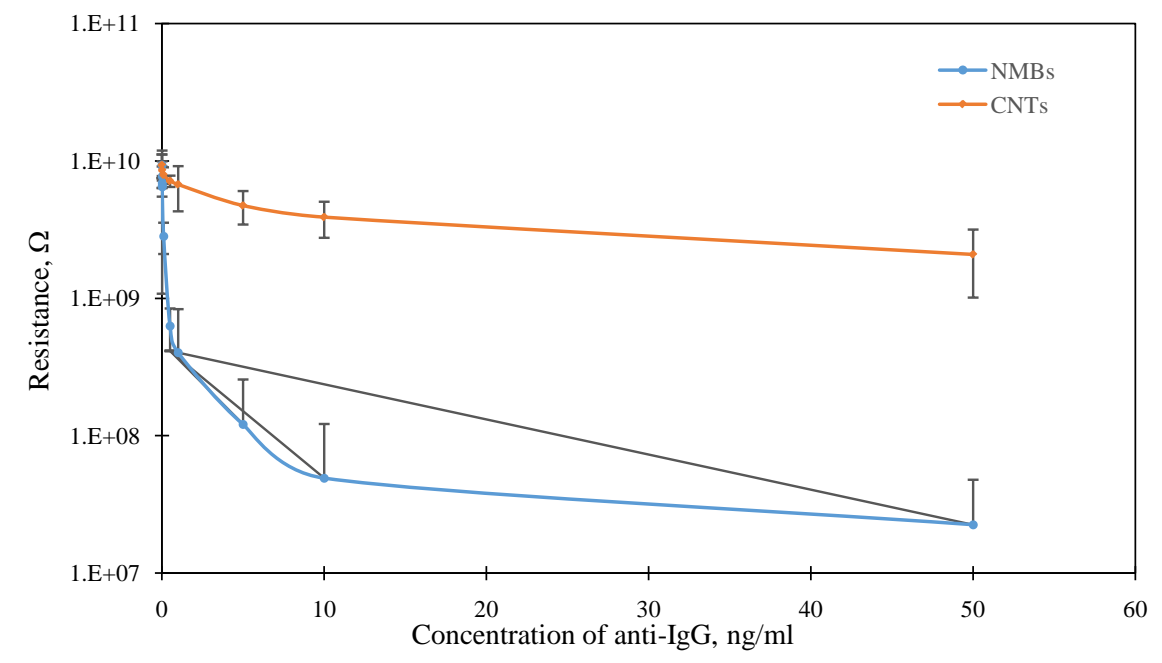

Figure 4. Resistivity of captured anti-IgG labeled with NMBs as compared to anti-IgG labeled with single walled carbon nanotubes. Each data point is the average of 5 measurements and the error bars represent the standard deviation. The limit of detection using NMBs was improved $\sim 3.45$ fold over the CNTs.

\section{Conclusion}

This study provided strong evidence that the use of carbon nanofibers grown on glass microballoons can be utilized to achieve ultra-low detection limit in rapid diagnostic test devices. Integration of millions of carbon nanofibers arranged on such micro-particle provided a higher probability of detecting minute concentrations of antigen in analyte. The NMBs also offered significantly higher level of sensitivity, as well as, simple and fast visual and/or electrical identification. Electrical resistivity measurements showed the potential for establishing a quantitative relationship for the antigen concentration. Using NMBs as label, ultra-low detection limits of $4 \mathrm{pg} / \mathrm{ml}$ for electrical signal and $0.1 \mathrm{mg} / \mathrm{ml}$ for visual detection were achieved. This novel approach holds the promise for early detection of various diseases, as well as providing healthcare professionals with information regarding the stage of infection. Utilizing this type of label could also lead to the elimination of false-alarms while reducing the incubation period required for detection in currently used RDTs. 


\section{Acknowledgements}

This research was supported by the National Science Foundation (Grant No. 0932300) under the Next Generation Composites CREST Center $\left(\mathrm{NextGenC}^{3}\right)$. We also acknowledge Dr. Ali Ghamsary and Dr. Ephraim Zegeye for their assistance.

\section{References}

[1] Chen, G. (2013) Fluorescence Biosensor for $\mathrm{H}_{5} \mathrm{~N}_{1}$ Antibody Based on Metal-Organic Framework Platform. Journal of Materials Chemistry B, 1, 1812-1817. http://dx.doi.org/10.1039/c3tb00501a

[2] Po-Yueh, W. and Lu, M.S.C. (2011) CMOS Thermal Sensor Arrays for Enzymatic Glucose Detection. Sensors Journal, IEEE, 11, 3469-3475. http://dx.doi.org/10.1109/JSEN.2011.2161283

[3] Rayana, R.R.-A., Hugo Javier, S.-P., María Liliana, M.-G., Bernardo, A.F.-U. and Abel, M. (2011) Chemical Biosensors Based on Proteins Involved in Biomineralization Processes. In: Serra, P.A., Ed., Biosensors—Emerging Materials and Applications, InTech, Gwalior.

http://www.intechopen.com/books/biosensors-emerging-materials-and-applications/chemical-biosensors-based-on-prot eins-involved-in-biomineralization-processes

[4] Lu, Y., Peng, S., Luo, D. and Lal, A. (2011) Low-Concentration Mechanical Biosensor Based on a Photonic Crystal Nanowire Array. Nature Communications, 2, 578. http://dx.doi.org/10.1038/ncomms1587

[5] Hua, W., Yan, C., Hassibi, A., Scherer, A. and Hajimiri, A. (2009) A Frequency-Shift CMOS Magnetic Biosensor Array with Single-Bead Sensitivity and No External Magnet. Solid-State Circuits Conference-Digest of Technical Papers, ISSCC 2009. IEEE International, 438-439.

[6] Zhang, D.-W., Liu, J.-X., Nie, J., Zhou, Y.-L. and Zhang, X.-X. (2013) Micropipet Tip-Based Miniaturized Electrochemical Device Combined with Ultramicroelectrode and Its Application in Immobilization-Free Enzyme Biosensor. Analytical Chemistry, 85, 2032-2036. http://dx.doi.org/10.1021/ac303223u

[7] Turner, A.P. (2013) Biosensors: Sense and Sensibility. Chemical Society Reviews, 42, 3184-3196. http://dx.doi.org/10.1039/c3cs35528d

[8] Childerhose, J.E. and Macdonald, M.E. (2013) Health Consumption as Work: The Home Pregnancy Test as a Domesticated Health Tool. Social Science \& Medicine, 86, 1-8. http://dx.doi.org/10.1016/j.socscimed.2013.02.035

[9] Lazcka, O., Campo, F.J.D. and Muñoz, F.X. (2007) Pathogen Detection: A Perspective of Traditional Methods and Biosensors. Biosensors and Bioelectronics, 22, 1205-1217. http://dx.doi.org/10.1016/j.bios.2006.06.036

[10] Linares, E.M., Kubota, L.T., Michaelis, J. and Thalhammer, S. (2012) Enhancement of the Detection Limit for Lateral Flow Immunoassays: Evaluation and Comparison of Bioconjugates. Journal of Immunological Methods, 375, $264-270$. http://dx.doi.org/10.1016/j.jim.2011.11.003

[11] Abera, A. and Choi, J.W. (2010) Quantitative Lateral Flow Immunosensor Using Carbon Nanotubes as Label. Analytical Methods, 2, 1819-1822. http://dx.doi.org/10.1039/c0ay00412j

[12] Fournier, P.E., Drancourt, M., Colson, P., Rolain, J.M., Scola, B.L., Raoult, D., Fournier, P.E., Drancourt, M., Colson, P., Rolain, J.M., Scola, B.L. and Raoult, D. (2013) Modern Clinical Microbiology: New Challenges and Solutions. Nature Reviews Microbiology, 11, 574-585. http://dx.doi.org/10.1038/nrmicro3068

[13] Justino, C.I.L., Rocha-Santos, T.A.P., Duarte, A.C. and Rocha-Santos, T.A.P. (2013) Advances in Point-of-Care Technologies with Biosensors Based on Carbon Nanotubes. TrAC Trends in Analytical Chemistry, 45, 24-36. http://dx.doi.org/10.1016/j.trac.2012.12.012

[14] Kim, J., Lee, J.Y., Jin, J.H., Park, C., Lee, C. and Min, N. (2012) A Fully Microfabricated Carbon Nanotube ThreeElectrode System on Glass Substrate for Miniaturized Electrochemical Biosensors. Biomedical Microdevices, 14, 613624. http://dx.doi.org/10.1007/s10544-012-9640-0

[15] Mendes, R.G., Bachmatiuk, A., Büchner, B., Cuniberti, G. and Rümmeli, M.H. (2013) Carbon Nanostructures as Multi-Functional Drug Delivery Platforms. Journal of Materials Chemistry B, 1, 401-428. http://dx.doi.org/10.1039/c2tb00085g

[16] Zegeye, E., Jin, Y. and Woldesenbet, E. (2012) A Paper Like Structure Formed by Binding Self-Assembled Glass Microballoons Using Random CNF Networks. Materials Letters, 68, 490-492. http://dx.doi.org/10.1016/j.matlet.2011.11.045

[17] Gikunoo, E., Abera, A. and Woldesenbet, E. (2014) A Novel Carbon Nanofibers Grown on Glass Microballoons Immunosensor: A Tool for Early Diagnosis of Malaria. Sensors, 14, 14686-14699. http://dx.doi.org/10.3390/s140814686

[18] Hermanson, G.T. (2008) Bioconjugate Techniques. 2nd Edition, Academic Press, London, 1323.

[19] Rao, V.K., Suresh, S., Sharma, M.K., Gupta, A. and Vijayaraghavan, R. (2011) Carbon Nanotubes-A Potential Ma- 
terial for Affinity Biosensors. Nanotechnology and Nanomaterials Carbon Nanotubes—Growth and Applications. InTech, Gwalior. http://www.intechopen.com/books/carbon-nanotubes-growth-and-applications

[20] Nguyen, L.Q., Phan, P.Q., Duong, H.N., Nguyen, C.D. and Nguyen, L.H. (2013) Enhancement of NH 3 Gas Sensitivity at Room Temperature by Carbon Nanotube-Based Sensor Coated with Co Nanoparticles. Sensors, 13, 1754-1762. http://dx.doi.org/10.3390/s130201754

[21] Marie, R., Beech, J.P., Vörös, J., Tegenfeldt, J.O. and Höök, F. (2006) Use of PLL-g-PEG in Micro-Fluidic Devices for Localizing Selective and Specific Protein Binding. Langmuir, 22, 10103-10108. http://dx.doi.org/10.1021/la060198m

[22] Chen, R.J., Bangsaruntip, S., Drouvalakis, K.A., Kam, N.W.S., Shim, M., Li, Y., Kim, W., Utz, P.J. and Dai, H. (2003) Noncovalent Functionalization of Carbon Nanotubes for Highly Specific Electronic Biosensors. Proceedings of the National Academy of Sciences of the United States of America, 100, 4984-4989. http://dx.doi.org/10.1073/pnas.0837064100

[23] Ding, Y., Li, D., Li, B., Zhao, K., Du, W., Zheng, J.Y. and Yang, M.H. (2013) A Water-Dispersible, Ferrocene-Tagged Peptide Nanowire for Amplified Electrochemical Immunosensing. Biosensors and Bioelectronics, 48, 281-286. http://dx.doi.org/10.1016/j.bios.2013.04.030

[24] Jie, G., Li, L.L., Chen, C., Xuan, J. and Zhu, J.J. (2009) Enhanced Electrochemiluminescence of CdSe Quantum Dots Composited with CNTs and PDDA for Sensitive Immunoassay. Biosensors and Bioelectronics, 24, 3352-3358. http://dx.doi.org/10.1016/j.bios.2009.04.039

[25] Liu, H., Wu, X.M., Zhang, X., Burda, C. and Zhu, J.J. (2011) Gold Nanoclusters as Signal Amplification Labels for Optical Immunosensors. The Journal of Physical Chemistry C, 116, 2548-2554. http://dx.doi.org/10.1021/jp206256j

[26] Leng, C., Wu, J., Xu, Q.N., Lai, G.S., Ju, H.X. and Yan, F. (2011) A Highly Sensitive Disposable Immunosensor through Direct Electro-Reduction of Oxygen Catalyzed by Palladium Nanoparticle Decorated Carbon Nanotube Label. Biosensors and Bioelectronics, 27, 71-76. http://dx.doi.org/10.1016/j.bios.2011.06.017

[27] Yang, Y.C., Dong, S.W., Shen, T., Jian, C.X., Chang, H.J., Li, Y. and Zhou, J.X. (2011) Amplified Immunosensing Based on Ionic Liquid-Doped Chitosan Film as a Matrix and Au Nanoparticle Decorated Graphene Nanosheets as Labels. Electrochimica Acta, 56, 6021-6025. http://dx.doi.org/10.1016/j.electacta.2011.04.096

[28] Liu, G., Chen, H.D., Peng, H.Z., Song, S.P., Gao, J.M., Lu, J.X., et al. (2011) A Carbon Nanotube-Based High-Sensitivity Electrochemical Immunosensor for Rapid and Portable Detection of Clenbuterol. Biosensors and Bioelectronics, 28, 308-313. http://dx.doi.org/10.1016/j.bios.2011.07.037

[29] Wang, Z., Gao, H. and Fu, Z. (2013) Introducing Novel Amorphous Carbon Nanoparticles as Energy Acceptors into a Chemiluminescence Resonance Energy Transfer Immunoassay System. Analyst, 138, 6753-6758. 
Scientific Research Publishing (SCIRP) is one of the largest Open Access journal publishers. It is currently publishing more than 200 open access, online, peer-reviewed journals covering a wide range of academic disciplines. SCIRP serves the worldwide academic communities and contributes to the progress and application of science with its publication.

Other selected journals from SCIRP are listed as below. Submit your manuscript to us via either submit@scirp.org or Online Submission Portal.
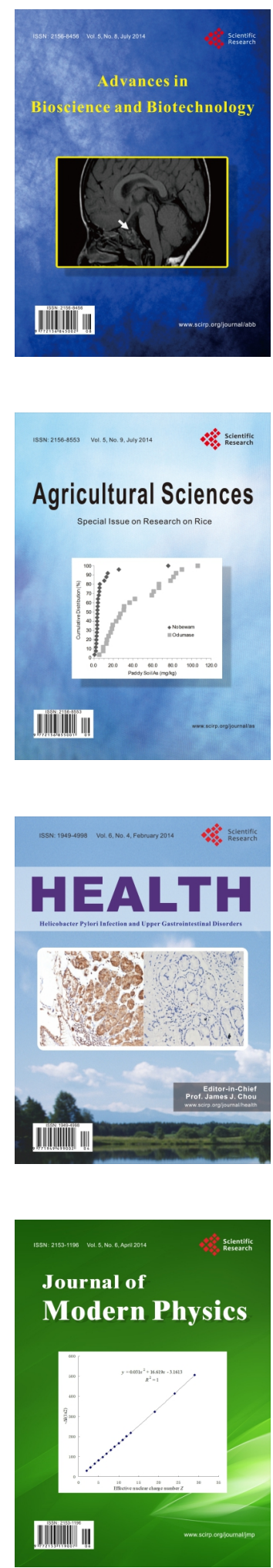
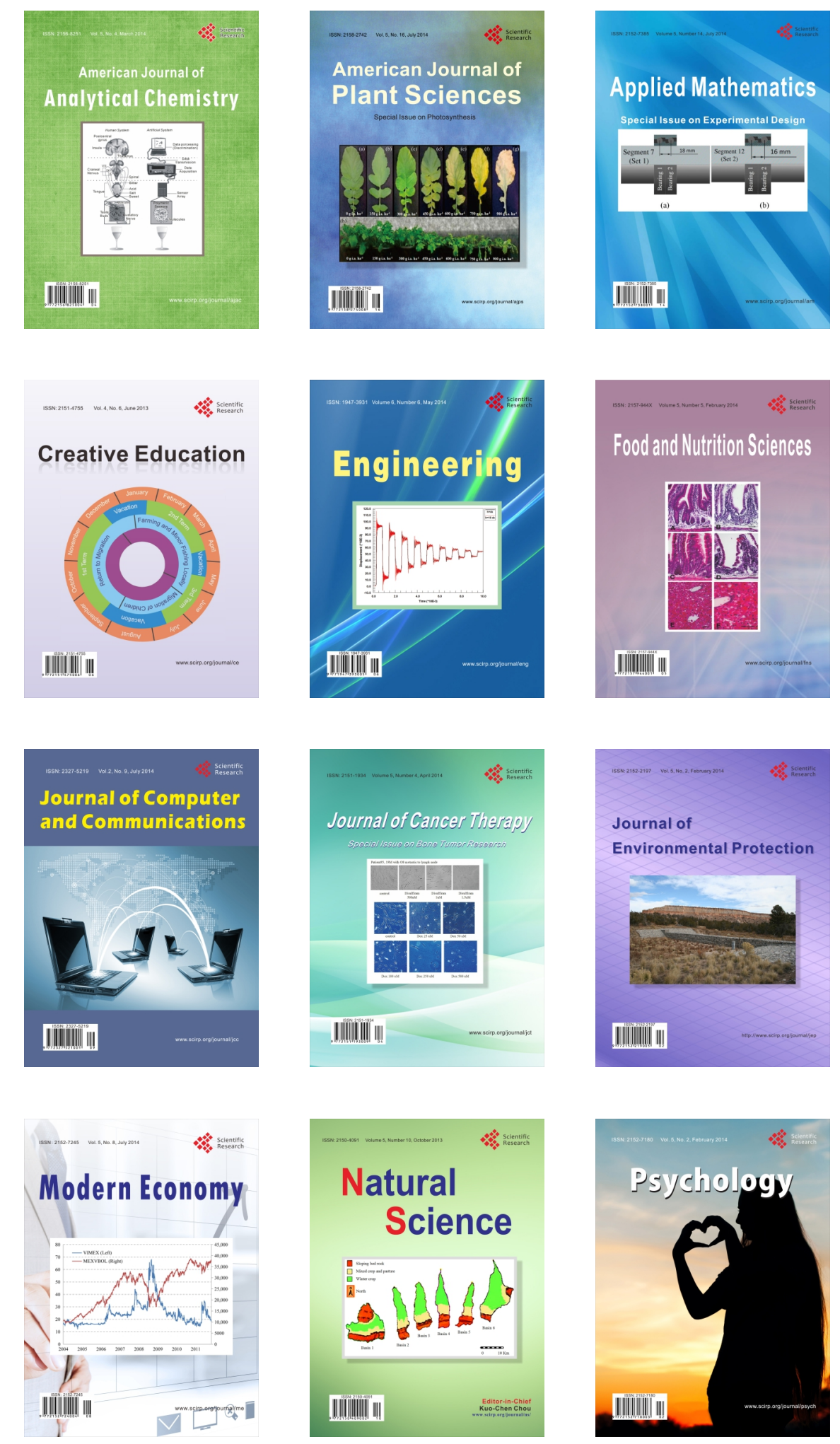